Research Article

\title{
The impact of three combinations vildagliptin/metformin, vildagliptin/ pioglitazone, and metformin/pioglitazone on glycemic control and atherogenic dyslipidemia in patients with Type 2 diabetes mellitus
}

\author{
Mandeep Kaur ${ }^{1,2 *}$, Jaswant Rai ${ }^{1}$, B. S. Bal ${ }^{3}$, Jasleen Kaur ${ }^{4}$
}

\begin{abstract}
${ }^{1}$ Department of Pharmacology, Government Medical College, Amritsar, Punjab, India, ${ }^{2}$ Department of Pharmacology, Adesh Institute of Medical Sciences \& Research, Bathinda, Punjab, India, ${ }^{3}$ Department of Medicine, Government Medical College, Amritsar, Punjab, India, ${ }^{4}$ Government Medical College, Amritsar, Punjab, India
\end{abstract}

Received: 20 August 2014 Accepted: 03 September 2014

*Correspondence to:

Dr. Mandeep Kaur, Email: mandeeplobana1@ yahoo.com

Copyright: (C) the author(s), publisher and licensee Medip Academy. This is an openaccess article distributed under the terms of the Creative Commons Attribution NonCommercial License, which permits unrestricted noncommercial use, distribution, and reproduction in any medium, provided the original work is properly cited.

\begin{abstract}
Background: There are limitations of currently recommended stepwise treatment for Type 2 diabetes, especially failure with monotherapies to achieve the strict glycemic control. This has prompted the intensification of therapy with such combinations which have additive efficacy and complimentary mechanisms of action. Vildagliptin is one such agent with the above potential which does not increase the risk of hypoglycemia and does not promote weight gain.

Methods: It was a prospective, open-label, randomized, and parallel group study involving 90 patients, divided into three Groups A, B, and C. Group A given vildagliptin/metformin (50/500 mg), Group B vildagliptin/pioglitazone (50/15 mg) and Group C metformin/pioglitazone (500/15 mg) combinations twice daily for 12 weeks. Fasting blood glucose (FBG) was estimated biweekly while hemoglobin $\mathrm{A} 1 \mathrm{c}(\mathrm{HbA} \mathrm{c})$ ), lipid profile, insulin, and C-peptide levels at 0 and $12^{\text {th }}$ week. Statistical analysis was done using ANOVA and Student's t-test.

Results: At the end, mean percentage of age fall in $\mathrm{HbAlc}$ and FBG from baseline was maximum in Group B, which was found out to be more efficacious than Group A and $\mathrm{C}(\mathrm{p}<0.001)$ on glycemic parameters. Mean percentage of age decrease in triglyceride from baseline was maximum in Group $\mathrm{C}$, which was found out to be more efficacious than Group A and B $(p<0.001)$ on lipid parameters. The adverse effects were low in all the groups. However, the incidence of peripheral edema and weight gain was more with the use of Group $\mathrm{C}$ while nausea, vomiting, and nasopharyngitis was more with the use of Group A.

Conclusion: Vildagliptin/pioglitazone combination is of choice in patients with uncontrolled hyperglycemia but normal lipid profile while metformin/pioglitazone combination in diabetic patients with dyslipidemia.
\end{abstract}

Keywords: C-peptide, Combination therapies, Diabetes mellitus, Incretin therapies, Insulin resistance

\section{INTRODUCTION}

Type 2 diabetes mellitus is a progressive disease characterized by insulin resistance (IR) and beta cell dysfunction. There is an urgent need to target both the defects to attain the ultimate goal of strict glycemic control and reduce the risk of morbidity and mortality associated with vascular and nonvascular complications accompanying the disease. According to American Diabetic Association (ADA) guidelines 2014, metformin should be the preferred initial pharmacological agent for Type 2 diabetes. The guidelines recommend the addition of other agents when noninsulin monotherapy at maximum tolerated dose does not achieve or maintain the hemoglobin $\mathrm{A} 1 \mathrm{c}(\mathrm{HbA} 1 \mathrm{c})$ target over 3 months. ${ }^{1}$ Since diabetes is a disease characterized by progressive deterioration of beta cell function, patients with monotherapy often fails to achieve the recommended glycemic control over time. Hence, there is a need of more 
aggressive initial therapy; specifically with early initiation of combination therapy.

Currently, various combination therapies are available for the management of diabetes which may provide an adequate glycemic control but at the expense of side effects such as hypoglycemia, weight gain, and edema. Thus, it becomes necessary to find out such combination therapies which have better safety profile, more effective in achieving the glycemic target $(\mathrm{HbA} 1 \mathrm{c}<7 \%)$ and address the underlying basic pathophysiology responsible for the progress of the disease. This highlights the importance of developing novel therapies that can overcome the shortcomings of conventional anti-diabetic medications. One such option is incretin-based therapies which offer a new approach to the management of Type 2 diabetes and vildagliptin is one such agent. This drug is a selective and reversible inhibitor of dipeptidyl peptidase-4 enzyme which is responsible for degradation of the incretin hormones (glucagon-like peptide-1 and glucose-dependent insulinotropic peptide) released by the gastrointestinal tract in response to a meal., ${ }^{2,3}$ These hormones stimulate the release of insulin from the pancreas which alone accounts for $50-70 \%$ of insulin secretion. ${ }^{4}$ Thus, vildagliptin effectively controls the blood sugar levels by increasing both $\alpha$ and $\beta$ cells responsiveness to glucose. ${ }^{5,6}$

Thus, the present research was undertaken to evaluate the clinical effectiveness and make head to head comparison between three combinations, i.e., vildagliptin/metformin, vildaglitin/pioglitazone, and metformin/pioglitazone in patients with Type 2 diabetes mellitus.

\section{METHODS}

\section{Study design}

This study was a randomized, prospective, open-label, and parallel conducted in accordance with the principles of good clinical practice and the declaration of Helsinki. The approval for the protocol of the study was sought from the Institutional Thesis and Ethical Committee. After obtaining approval from the concerned authorities, the study was initiated, and patients were recruited after seeking their informed consent. The patients were advised to bring the empty blister packs of the medications at every visit to ensure consent in accordance with the established protocol, compliance with medication, and to report immediately on experiencing any adverse event during the study period.

\section{Patient selection}

The study enrolled patients of Type 2 diabetes mellitus who were on metformin or pioglitazone monotherapy with inadequate glycemic control and in the age group of 35-70 years, with glycosylated hemoglobin levels $>7.0 \%$. Patients were excluded from the present study who: presented with disorders like angina, cardiac insufficiency, cardiac failure (NYHA III-IV), pre-existing hepatic illness (aspartate aminotransferase, alanine aminotransferase levels $>2.5$ times normal), diabetic ketoacidosis, nephropathy, chronic renal failure, advanced retinopathy; women pregnant or lactating; on drugs like statins, fibrates or other antilipemic agents; were requiring insulin therapy. The patients were advised to continue with their dietary modification and physical activity, and they were explained the schedule of the drug treatment. The patients were also advised to report immediately in case they developed hypoglycemia or any other symptoms pertaining to side effects of the drugs. At the end of the study, the patients were kept under regular observation and those who require insulin were excluded from the study but were managed with other available drugs.

\section{Treatment}

Patients were randomized into three groups with Group A receiving vildagliptin and metformin combination (50/500 mg), Group B vildagliptin and pioglitazone combination $(50 / 15 \mathrm{mg})$ and Group $\mathrm{C}$ metformin and pioglitazone combination (500/15 mg). In each group, drug was administered twice daily for 12 weeks.

\section{Study assessments}

The patients were instructed to visit the hospital in the morning after overnight fasting for $12 \mathrm{hrs}$. A history of the patient, clinical examination, and adverse events were recorded, and blood samples were taken following aseptic technique.

Estimation of HbA1c, total cholesterol (TC), low-density lipoprotein (LDL), high-density lipoprotein (HDL) and triglyceride (TG), fasting plasma insulin levels (FPI), and C-peptide levels were done at 0 week and $12^{\text {th }}$ week of the study. Fasting blood glucose (FBG) was monitored at bi-weekly interval throughout the study. The IR was calculated by the formula: ${ }^{7}$

$$
\mathrm{IR}=\frac{\mathrm{FPI} \times \mathrm{FBG}}{22.5}
$$

$\mathrm{FPI}=\mu \mathrm{IU} / \mathrm{ml}$

$\mathrm{FBG}=\mathrm{mmol} / \mathrm{dl}$

Estimation of:

1. FBS done by glucose oxidase method using glucose reagent and glucose standard

2. HbA1c done by cation exchange resin method using lysing reagent, ion exchange, resin control, and resin separators

3. FPI and C-peptide levels done by monobind insulin microplate ELISA test (accubind ELISA Microwells, Monobind Inc.) 
4. Serum TC done by enzymatic method using TC kit (monozyme) enzyme reagents and cholesterol standard

5. HDL-cholesterol (HDL-C) levels done by the phosphotungstate method using enzyme reagents, cholesterol standard, precipitating reagent

6. TG levels done by enzymatic method using TG kit (lyphozyme) using enzyme reagents and the glycerol standard

7. LDL-C calculated using Friedewald's formula:

LDL-C $(\mathrm{mg} / \mathrm{dl})=\mathrm{TC}(\mathrm{mg} / \mathrm{dl})-\mathrm{HDL}-\mathrm{C}(\mathrm{mg} / \mathrm{dl})-\mathrm{TG}(\mathrm{mg} / \mathrm{dl}) / 5$.

\section{Statistical analysis}

A validated statistical software GraphPad StatMate 2.00 (GraphPad Software) (http://www.graphpad.com/statmate/ upgrade.htm) was used for the analyses and graphical representation of the data. ANOVA was used to compare the observations of all the three groups at the end points while Student's t-test was used to compare the end point values with the baseline in individual group.

\section{RESULTS}

\section{Study patients}

Out of 115 patients screened, 102 were found eligible to be included in the study and were randomized to Group A, Group B, and Group C. All the randomized patients received at least one dose of the study medication. A total of 90 patients completed the study. The disposition of the patients is provided in Figure 1. The confirmatory results described here are based on the per-protocol (PP) population. Baseline characteristics of the patients are presented in Table 1. There were no significant differences between groups for these parameters $(p>0.05)$. The primary efficacy endpoint was the change from baseline to endpoint in $\mathrm{HbA} 1 \mathrm{c}, \mathrm{FBG}, \mathrm{FPI}$, C-peptide, and lipid profile in the PP population.

\section{Effect on glycemic parameters}

Glycemic parameters included the evaluation of $\mathrm{HbA} 1 \mathrm{c}$, FBG, FPI, C-peptide levels, and IR which were comparable at baseline.

A significant fall in FBG in all the three groups at the end of the study $(\mathrm{p}<0.001)$ was observed with Group B showing greater reduction (Table 2 ). The mean percentage reduction in Group B (25.94\%) was more as compared to Group A (18.10\%) and Group C (19.03\%). This mean percentage reduction in Group B is significant in comparison to both Group A and Group C. Similarly, a significant fall in HbA1c was observed from baseline in all the three groups $(p<0.001)$. There is a greater reduction of HbA1c in Group B shown in Table 2. The mean percentage reduction in Group B (15.72\%) was more as compared to Group A (8.88\%) and Group C $(9.87 \%)$. This mean percentage reduction is significant in Group B in comparison to both Group A and Group C.

At the end of 12 weeks, there was a significant decrease $(p<0.001)$ in FPI levels in all the three groups (Table 2). The maximum fall in mean \% age of 13.12 in FPI was observed in Group B with minimum fall of 0.82 in Group A. There was significant decrease in fasting $\mathrm{C}$-peptide levels in Group B and Group C $(\mathrm{p}<0.001)$ and insignificant decrease in Group A ( $>>0.05)$ (Table 2). The maximum fall in mean $\%$ age of 13.73 in C-peptide was observed in Group B with a minimum fall of 0.96 in Group A.

A significant decrease in IR was observed in all the three groups at the end of the study $(\mathrm{p}<0.001)$ (Table 2) while, in Group B, the percentage decrease was more $(35.73 \%)$ in comparison to Group A (18.76\%) and C (28.75\%).

\section{Effect on lipid parameters}

Lipid parameters included the evaluation of fasting TG, HDL, TC, and LDL cholesterol which were comparable at baseline.

Table 1: Baseline characteristics of patients.

\begin{tabular}{|c|c|c|c|}
\hline Characteristic & Group A & Group B & Group C \\
\hline Number of patients & 30 & 30 & 30 \\
\hline Mean age (years) & $54.87 \pm 6.96$ & $57.50 \pm 8.99$ & $56.60 \pm 9.514$ \\
\hline $\operatorname{Sex}(M: F)$ & $10: 20$ & $12: 18$ & $11: 19$ \\
\hline HbA1c (\%) & $8.43 \pm 0.75$ & $8.56 \pm 0.69$ & $8.55 \pm 0.84$ \\
\hline $\mathrm{FBG}(\mathrm{mg} / \mathrm{dl})$ & $168.70 \pm 19.54$ & $177.87 \pm 16.4$ & $169.53 \pm 19.53$ \\
\hline Fasting insulin $(\mu \mathrm{IU} / \mathrm{ml})$ & $24.46 \pm 3.34$ & $23.08 \pm 4.83$ & $23.14 \pm 6.55$ \\
\hline HOMA-IR & $10.17 \pm 1.74$ & $9.99 \pm 1.52$ & $9.53 \pm 2.40$ \\
\hline Fasting C-peptide (ng/ml) & $2.93 \pm 0.40$ & $2.71 \pm 04.71$ & $2.84 \pm 0.43$ \\
\hline $\mathrm{TG}(\mathrm{mg} / \mathrm{dl})$ & $192.43 \pm 23.45$ & $185.27 \pm 27.43$ & $191.13 \pm 36.60$ \\
\hline HDL-C (mg/dl) & $37.76 \pm 4.07$ & $36.76 \pm 2.56$ & $38.01 \pm 5.354$ \\
\hline $\mathrm{TC}(\mathrm{mg} / \mathrm{dl})$ & $190.07 \pm 21.17$ & $187.67 \pm 13.49$ & $194.93 \pm 19.93$ \\
\hline LDL-C (mg/dl) & $113.82 \pm 22.41$ & $113.85 \pm 10.70$ & $118.70 \pm 16.95$ \\
\hline
\end{tabular}

TC: Total cholesterol, LDL-C: Low density lipoprotein-cholesterol, HDL-C: High density lipoprotein-cholesterol, TG: Triglyceride 


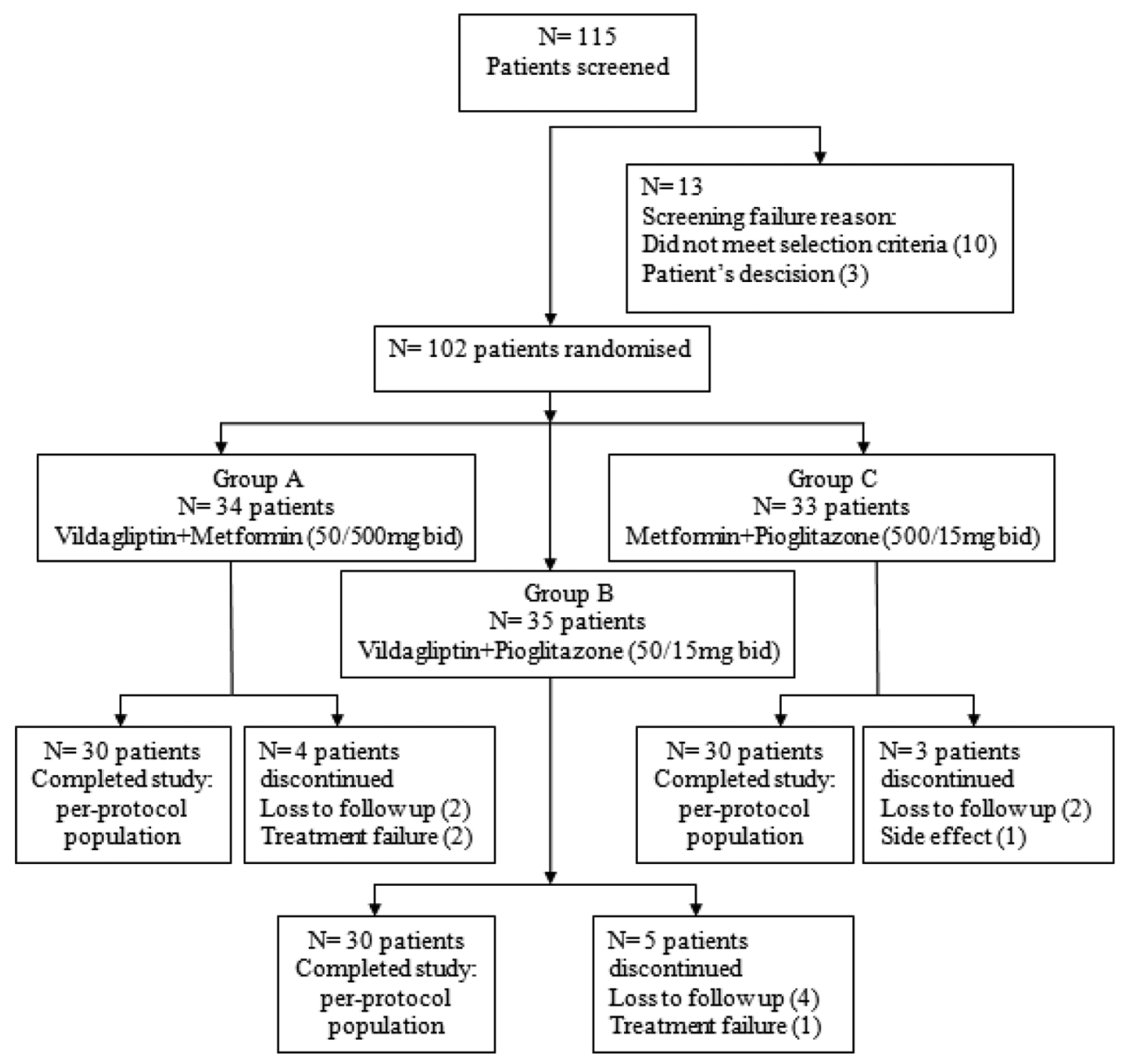

Figure 1: Disposition of patients.

Table 2: Effect on glycemic parameters.

\begin{tabular}{|lccc|}
$\begin{array}{l}\text { Time period } \\
\text { (weeks)/ }\end{array}$ & Group A & Group B & Group C \\
parameters & & & \\
\hline FBG (mg/dl) & & & \\
\hline 0 & $168.70 \pm 19.54$ & $177.87 \pm 16.40$ & $169.53 \pm 19.53$ \\
\hline 12 & $137.17 \pm 8.47$ & $131.07 \pm 8.28$ & $136.20 \pm 7.94$ \\
\hline HbA1c \% & & & \\
\hline 0 & $8.43 \pm 0.75$ & $8.56 \pm 0.69$ & $8.55 \pm 0.84$ \\
\hline 12 & $7.68 \pm 0.69$ & $7.21 \pm 0.70$ & $7.70 \pm 0.70$ \\
\hline FPI ( $\mu$ IU/ml) & & & \\
\hline 0 & $24.46 \pm 3.28$ & $23.08 \pm 4.83$ & $23.14 \pm 6.55$ \\
\hline 12 & $24.26 \pm 3.34$ & $20.18 \pm 4.78$ & $20.67 \pm 6.90$ \\
\hline $\begin{array}{l}\text { C-peptide } \\
\text { (ng/ml) }\end{array}$ & & & \\
\hline 0 & $2.93 \pm 0.40$ & $2.71 \pm 0.471$ & $2.84 \pm 0.43$ \\
\hline 12 & $2.89 \pm 0.42$ & $2.36 \pm 0.48$ & $2.54 \pm 0.47$ \\
\hline IR & & & \\
\hline 0 & $10.17 \pm 1.74$ & $9.99 \pm 1.52$ & $9.53 \pm 2.40$ \\
\hline 12 & $8.21 \pm 1.24$ & $6.46 \pm 1.32$ & $6.90 \pm 2.19$ \\
\hline
\end{tabular}

IR: Insulin resistance, FPI: Fasting plasma insulin,

FBG: Fasting blood glucose, HbA1c: Hemoglobin A1c
Mean fasting TG levels decreased significantly $(\mathrm{p}<0.001)$ in all the three groups, at the end of the study with the highest fall observed in Group C (Table 3). The adjusted mean percentage of change in TG was $4.27 \%, 9.13 \%$, $13.36 \%$ in Group A, B, and C respectively. Mean fasting HDL levels increased significantly in all the three groups (Table 3 ) at the end of the study with adjusted mean percentage of change of $4.45,11.38,15.89 \%$ in Group A, $\mathrm{B}$, and $\mathrm{C}$, respectively. The changes in TG and HDL were statistically significant in Group $\mathrm{C}$ in comparison to other groups.

A highly significant $(\mathrm{p}<0.001)$ decrease in TC in group A, insignificant $(\mathrm{p}>0.05)$ decrease in Group B and a significant increase in Group C $(p<0.01)$ was observed at the end of the study (Table 3 ). The adjusted mean percentage of change in TC was 2.21, 0.48 and $1.21 \%$ in Group A, B, and C, respectively.

A significant decrease in LDL in both the Groups A and $B$ and insignificant $(\mathrm{p}>0.05)$ increase in Group C were observed (Table 3 ). The adjusted mean percentage of change in LDL was 3.79, 1.42, and $1.56 \%$ in Group A, B, and C, respectively. 
Table 3: Effect on lipid parameters.

\begin{tabular}{|llll|}
$\begin{array}{l}\text { Time period } \\
\text { (weeks)/ } \\
\text { parameters }\end{array}$ & Group A & Group B & Group C \\
\hline TG $(\mathrm{mg} / \mathrm{dl})$ & & & \\
\hline 0 & $192.43 \pm 23.45$ & $185.27 \pm 27.43$ & $191.13 \pm 36.60$ \\
\hline 12 & $184.13 \pm 21.94$ & $168.40 \pm 26.03$ & $165.87 \pm 35.03$ \\
\hline HDL $(\mathrm{mg} / \mathrm{dl})$ & & & \\
\hline 0 & $37.76 \pm 4.07$ & $36.76 \pm 2.56$ & $38.01 \pm 5.35$ \\
\hline 12 & $39.38 \pm 3.91$ & $40.87 \pm 3.03$ & $43.76 \pm 4.48$ \\
\hline TC $(\mathrm{mg} / \mathrm{dl})$ & & & \\
\hline 0 & $190.07 \pm 21.17$ & $187.67 \pm 13.49$ & $194.93 \pm 19.93$ \\
\hline 12 & $185.90 \pm 21.57$ & $186.73 \pm 13.31$ & $197.27 \pm 20.33$ \\
\hline LDL $(\mathrm{mg} / \mathrm{dl})$ & & & \\
\hline 0 & $113.82 \pm 22.41$ & $113.85 \pm 10.70$ & $118.70 \pm 16.95$ \\
\hline 12 & $109.65 \pm 22.92$ & $112.19 \pm 10.96$ & $120.33 \pm 16.21$ \\
\hline
\end{tabular}

TC: Total cholesterol, LDL: Low density lipoprotein,

HDL: High density lipoprotein, TG: Triglyceride

\section{Tolerability}

The side effect profile was comparable in all the three groups and no serious events required the discontinuation of therapy occurred in any group. However, the incidence of peripheral edema and weight gain was more in metformin/pioglitazone group as compared to other groups while nausea, vomiting, and nasopharyngitis was more with the use of metformin/ vildagliptin group.

\section{DISCUSSION}

The results from this study showed that the use of vildagliptin/metformin, vildagliptin/pioglitazone, and metformin/pioglitazone combination in patients with Type 2 diabetes showed a significant fall in $\mathrm{HbA1c}, \mathrm{FBG}$, and IR $(p<0.001)$ with respect to baseline but this fall was maximum and significant in vildagliptin/pioglitazone combination group when compared to others. This maximum fall may be due to their complementary mechanism of action as vildagliptin enhances the pancreatic islet cell function ${ }^{6}$ and pioglitazone decreases the IR. ${ }^{8}$ It was also found out at the end of study that more number of patients in vildagliptin/ pioglitazone combination group were able to achieve ADA recommended target of $\mathrm{HbA} 1 \mathrm{c}<7 \%$ and $\mathrm{FBG}<130 \mathrm{mg} / \mathrm{dl}$ when compared to other two groups (Table 4).

The present research work has also demonstrated that vildagliptin/metformin and metformin/pioglitazone combination were equally effective in controlling the FBG and HbA1c levels. At the end of 12 weeks, when compared to baseline, reduction in mean percentage of FBG of $18.10 \pm 5.98$ and $\mathrm{HbAlc}$ of $8.88 \pm 3.23$ with the use of vildagliptin/metformin combination and reduction in mean percentage of FBG of $19.03 \pm 6.25$ and $\mathrm{HbA} 1 \mathrm{c}$ of $9.87 \pm 3.15$ with the use of metformin/pioglitazone combination were
Table 4: Percentage of patients achieved ADA target.

\begin{tabular}{|lccc|}
\hline Parameters & Group A & Group B & Group C \\
\hline FBG $(<130 \mathrm{mg} / \mathrm{dl})$ & 33.33 & 53.3 & 36.6 \\
\hline HbA1c $(<7 \%)$ & 30 & 50 & 26.67 \\
\hline TG $(<150 \mathrm{mg} / \mathrm{dl})$ & 6.66 & 23.33 & 36.66 \\
\hline HDL $(>50 \mathrm{mg} / \mathrm{dl})$ & 43.33 & 73.33 & 76.67 \\
\hline LDL $(<100 \mathrm{mg} / \mathrm{dl})$ & 43.33 & 10 & 13.33 \\
\hline
\end{tabular}

LDL: Low density lipoprotein, HDL: High density

lipoprotein, TG: Triglyceride, FBG: Fasting blood glucose, HbA1c: Hemoglobin Alc

observed $(p>0.05)$. These study results were consistent with the results of Bolli et al. study ${ }^{9}$ showing vildagliptin to be non-inferior to pioglitazone when added to metformin.

Metformin/pioglitazone combination showed a statistically significant improvement in TG and HDL levels with more number of patients able to achieve ADA recommended target of $\mathrm{TG}<150$ and $\mathrm{HDL}>50 \mathrm{mg} / \mathrm{dl}$ in comparison to other two combinations which has also been reported in other studies. ${ }^{1,10-13}$ However, our combination showed a modest increase in LDL and TC levels which is in accordance with the study done by Perenz et al. ${ }^{10}$ who also reported an increase in LDL particle size with the use of this combination which probably reduces the risk of atherosclerosis. The use of vildgliptin/metformin combination showed highly significant improvement $(\mathrm{p}<0.001)$ in LDL and TC levels with more number of patients achieving the ADA recommended target of $\mathrm{LDL}<100 \mathrm{mg} / \mathrm{dl}^{1}$ than the other two combinations which confirmed the results reported by Bolli et al. ${ }^{9}$

Vildagliptin/metformin combination caused nausea and vomiting in 3 patients $(10 \%)$, nasopharyngitis and headache in one patient (3.3\%). Vildagliptin/pioglitazone combination caused headache and hypoglycemia in one patient (3.33\%) while metformin/pioglitazone combination caused nausea, vomiting, and hypoglycemia in one patient $(3.3 \%)$, peripheral edema, and weight gain in two patients $(6.6 \%)$. No gain in body weight and edema was experienced by patients with vildagliptin/metformin and vildagliptin/pioglitazone combination. This is in accordance with the Bolli et al. study. ${ }^{9}$ These side effects were not serious enough either to discontinue the therapy or to hospitalize the patients. Thus, overall vildagliptin/pioglitazone combination was found out to be safer than the other two combinations.

\section{CONCLUSION}

In the present study, vildagliptin/pioglitazone combination lower the FBS, HbA1c, and IR more effectively and thus can be used in diabetic patients with uncontrolled hyperglycemia on metformin monotherapy without increasing the incidence of side effects. In contrast, metformin/pioglitazone combination provides better control of TG and HDL levels in addition to glycemic control which is equivalent to vildagliptin/ metformin combination. Thus, use of this combination is of choice in diabetic patients with dyslipidemia. 
Funding: None

Conflict of interest: None declared

Ethical approval: The study was approved by the Institutional

Ethics Committee

\section{REFERENCES}

1. American Diabetic Association (ADA) 2014 guidelines. Available at http://www.ndei.org/ADA-2014-guidelinesdiabetes-diagnosis-A1C-testing.aspx. Accessed 16 Aug 2014.

2. Babu S. Vildagliptin - A new prospect in management of type 2 diabetes. Indian J Clin Pract. 2012;22:377-85.

3. Stonehouse A, Okerson T, Kendall D, Maggs D. Emerging incretin based therapies for type 2 diabetes: incretin mimetics and DPP-4 inhibitors. Curr Diabetes Rev. 2008;4(2):101-9.

4. Balas B, Baig MR, Watson C, Dunning BE, Ligueros-Saylan M, Wang Y, et al. The dipeptidyl peptidase IV inhibitor vildagliptin suppresses endogenous glucose production and enhances islet function after single-dose administration in type 2 diabetic patients. J Clin Endocrinol Metab. 2007;92(4):1249-55.

5. Ahrén B, Landin-Olsson M, Jansson PA, Svensson M, Holmes D, Schweizer A. Inhibition of dipeptidyl peptidase-4 reduces glycemia, sustains insulin levels, and reduces glucagon levels in type 2 diabetes. J Clin Endocrinol Metab. 2004;89(5):2078-84.

6. Mari A, Sallas WM, He YL, Watson C, Ligueros-Saylan M, Dunning BE, et al. Vildagliptin, a dipeptidyl peptidase-IV inhibitor, improves model-assessed beta-cell function in patients with type 2 diabetes. J Clin Endocrinol Metab. 2005;90(8):4888-94.

7. Matthews DR, Hosker JP, Rudenski AS, Naylor BA, Treacher DF, Turner RC. Homeostasis model assessment: insulin resistance and beta-cell function from fasting plasma glucose and insulin concentrations in man. Diabetologia. 1985;28(7):412-9.
8. Roden M, Laakso M, Johns D, Widel M, Urquhart R, Richardson C, et al. Long-term effects of pioglitazone and metformin on insulin sensitivity in patients with Type 2 diabetes mellitus. Diabet Med. 2005;22(8):1101-6.

9. Bolli G, Dotta F, Rochotte E, Cohen SE. Efficacy and tolerability of vildagliptin vs. pioglitazone when added to metformin: a 24-week, randomized, double-blind study. Diabetes Obes Metab. 2008;10(1):82-90.

10. Perez A, Khan M, Johnson T, Karunaratne M. Pioglitazone plus a sulphonylurea or metformin is associated with increased lipoprotein particle size in patients with type 2 diabetes. Diab Vasc Dis Res. 2004;(1):44-50.

11. Rosenstock J, Einhorn D, Hershon K, Glazer NB, Yu S. Pioglitazone 014 Study Group. Efficacy and safety of pioglitazone in type 2 diabetes: a randomised, placebocontrolled study in patients receiving stable insulin therapy. Int J Clin Pract. 2002;56(4):251-7.

12. Einhorn D, Rendell M, Rosenzweig J, Egan JW, Mathisen AL, Schneider RL. Pioglitazone hydrochloride in combination with metformin in the treatment of type 2 diabetes mellitus: a randomized, placebo-controlled study. The Pioglitazone 027 Study Group. Clin Ther. 2000;22(12):1395-409.

13. Kipnes MS, Krosnick A, Rendell MS, Egan JW, Mathisen AL, Schneider RL. Pioglitazone hydrochloride in combination with sulfonylurea therapy improves glycemic control in patients with type 2 diabetes mellitus: a randomized, placebo-controlled study. Am J Med. 2001;111(1):10-7.

doi: $10.5455 / 2319-2003$. ijbcp20141036

Cite this article as: Kaur M, Rai J, Bal BS, Kaur J. The impact of three combinations vildagliptin/metformin, vildagliptin/pioglitazone, and metformin/pioglitazone on glycemic control and atherogenic dyslipidemia in patients with Type 2 diabetes mellitus. Int J Basic Clin Pharmacol 2014;3:902-7. 